\title{
Çok Uzun Baz Enterferometrisi (VLBI) ölçülerinin modellenmesi ${ }^{*}$
}

\author{
Kamil Teke ${ }^{1, *}$, Emine Tanır Kayıkçı ${ }^{2}$, Johannes Böhm³ ${ }^{3}$, Harald Schuh ${ }^{3}$ \\ ${ }^{1}$ Hacettepe Üniversitesi, Mühendislik Fakültesi, Geomatik Mühendisliği Bölümü, 06800, Ankara, Türkiye \\ ${ }^{2}$ Karadeniz Teknik Üniversitesi, Mühendislik Fakültesi, Harita Mühendisliği Bölümü, 61080, Trabzon, Türkiye \\ ${ }^{3}$ Viyana Teknoloji Üniversitesi, Jeodezi ve Jeofizik Enstitüsü, 1040, Viyana, Avusturya

\section{Özet}

Web Yayın: 10 Temmuz 2012 Bu çalışmanin temel amacı Çok Uzun Baz Enterferometrisi (VLBI) sinyal gecikme modelinin teorik detaylarını Uluslararası Yer Dönüklük ve Referans Sistemleri Servisi (IERS) 2010 Konvansiyonları temelinde sunmaktır. Bu kapsamda sürekli parçalı lineer ofset (CPWLO) fonksiyonları ile gün-içi zamansal çözünürlükte önceden belirlenmiş epoklarda, örneğin: Evrensel Zaman (UT) tam saatleri, tam saatlerin katlarl veya bölümlerinde jeodezik parametrelerin kestirimi ayrıca açıklanmıştır. Yerküre'deki homojen dağılımlı Yer-sabit VLBI antenlerinden gök küredeki homojen dağllımlı uzay-sabit radyo kaynaklarına her parametre kestirim epoğu aralı̆̆ında yapılacak yeterli sayıda VLBI ölçülerinden, jeodezik parametreler gün-içi zamansal çözünürlükte eşzamanlı ve duyarlı̆̆ yüksek olarak kestirilebilir. Saat senkronizasyon ve troposfer gecikme modellerini içeren temel VLBI gecikme modeli sunulduktan sonra VLBI ölçü denkleminin başlıca jeodezik parametrelere göre kısmi türevleri verilmiş ve bazı VLBI sonuçları gösterilmiştir.

Anahtar Sözcükler

Çok Uzun Baz Enterferometrisi (VLBI), VLBI gecikme modeli, sürekli parçalı lineer ofsetler, CPWLO.

Pub. Online: 10 July 2012

Volume: 1

Number: 1

Page: 65 - 74

May 2012

\section{Abstract}

\section{Modelling Very Long Baseline Interferometry (VLBI) observations}

The main objective of this study is to provide in detail the theoretical basis of the Very Long Baseline Interferometry (VLBI) delay model, mainly according to the International Earth Rotation and Reference Systems Service (IERS) Conventions 2010. This goes along with introducing the concept of continuous piece-wise linear offset (CPWLO) functions for estimating sub-daily geodetic parameters at pre-defined epochs, e.g. at Universal Time (UT) integer hours or at integer fractions or multiples of integer hours. The geodetic parameters can be simultaneously and accurately estimated from VLBI observations in sub-daily resolution if enough observations within each estimation interval are carried out from homogenously distributed Earth-fixed VLBI antennas to space-fixed radio sources. After providing the basic VLBI model of the geometric delay including clock synchronization and tropospheric effects, the partial derivatives of VLBI observation equation with respect to the most important geodetic parameters are given and some typical $V L B I$ results are shown.

Keywords

Very Long Baseline Interferometry (VLBI), VLBI delay model, continuous piece-wise linear offsets, CPWLO. 


\section{Giriş}

Çok Uzun Baz Enterferometrisi (Very Long Baseline Interferometry, VLBI) tekniği yaklaşık 40 yıldır jeodezik amaçlar doğrultusunda kullanılmaktadır. 1999 yılından bu yana Uluslararası Jeodezi ve Astrometri VLBI Servisi (International VLBI Service for Geodesy and Astrometry, IVS) Uluslararası Astronomi Birliği’nin (International Astronomical Union, IAU) ve Uluslararası Jeodezi Kuruluşu'nun (International Association of Geodesy, IAG) bir servisidir (Schlüter ve Behrend 2007). VLBI tekniğinin bilimin hizmetine sunduğu başlıca veriler ve ürünler; Yer-sabit referans sisteminde (Uluslararası Yersel Referans Sistemi, International Terrestrial Reference System, ITRS) tanımlı gök ortalama kutbu (Celestial Intermediate Pole, CIP) konumu (kutup gezinmesi koordinatları), Yer'in ortalama dönme ekseninin uzay-sabit referans sistemine (Yer-Merkezli Göksel Referans Sistemi, Geocentric Celestial Reference System, GCRS) göre mutlak dönüklüğü (UT1-UTC), gök-sabit referans sisteminde (Uluslararası Göksel Referans Sistemi, International Celestial Reference System, ICRS) tanıml CIP konumu (nutasyon ofsetleri), Güneş Sistemi Merkezli Göksel Referans Sisteminde (Barycentric Celestial Reference System, BCRS) tanımlı radyo kaynağı (quasi-stellar radio source, quasar (kuazar)) konumu, ITRS'de tanımlı anten konumu, iyonosfer sinyal gecikmeleri, yükselim açısına göre değişen troposfer sinyal gecikmeleri, azimut açısına göre değişen troposfer sinyal gecikmeleri (troposfer gradyanları), saat hataları, güneş, ay ve diğer güneş sistemindeki gezegenlerinin Yer'in dış gravite alanı içerisinde oluşturduğu gel-git (tidal) potansiyeline Yer'in verdiği visko-elastik radyal ve yatay tepki katsayıları: love ve shida numaraları ve teknik (VLBI) özel parametreler, şeklinde sıralanabilir.

Çok Uzun Baz Enterferometrisi (VLBI) geometrik bir uzay jeodezisi tekniğidir. VLBI galaksimiz dışındaki radyo kaynaklarından dünyaya ulaşan radyo dalgasının iki Yer-sabit antene ulaşmasındaki zaman gecikmesini ölçer (Şekil 1).

Bir çok kuazardan dünyamıza ulaşan radyo dalgalarının Yer'i saran global ağı oluşturan antenlerin algıladığı varış



Şekil 1: VLBI ölçü prensibi: Temel ölçü galaksi dışındaki bir radyo kaynağından gönderilen ve dünyaya düzlem bir yüzey formunda çarpan radyo dalgasının iki VLBI teleskobuna ulaşımındaki zaman gecikmesidir. zamanları gecikmeleri ölçülerinden, VLBI; inersiyal referans çatısını (uzay-sabit çatı), örneğin ICRF2 (Fey vd. 2009), eş zamanlı olarak Yersel referans çatısını (yer-sabit çatı), belirler. VLBI teknolojisi ile ilgili detaylı bilgi Campbell (1979, 2004), Schuh (1987), Nothnagel (1991), Sovers vd. (1998), Takahashi vd. (2000), Böhm vd. (2011a), Teke vd. (2009) ve Teke (2011) kaynaklarından edinilebilir.

Makalenin 2. Bölümü'nde "uzlaşılan model" olarak bilinen ve Uluslararası Yer Dönüklük ve Referans Sistemleri Servisi (International Earth Rotation and Reference Systems Service, IERS) tarafından önerilen VLBI gecikme modeli açıklanmıştır. Bizler temel olarak anten koordinatlarının Uluslararası Yersel Referans Sisteminden (ITRS) YerMerkezli Göksel Referans Sistemine (GRCS) dönüşümünde ve VLBI gecikme modelinin oluşturulmasında IERS 2010 konvansiyonlarını (Petit ve Luzum 2010) esas aldık. 2.3 ve 2.4 alt bölümleri sırası ile saat ve troposfer hatalarından kaynaklanan sinyal gecikme modellerini tanıtmıştır. 2.5 alt bölümünde VLBI gecikme modelinin bazı kestirimi yapılan parametrelere göre kısmi türevleri Sovers vd. (1998), Böhm vd. (2011a) ve Teke (2011) temelinde verilmektedir. Bölüm 3 'de ise gün-içi zamansal çözünürlükte VLBI parametrelerinin kestirimini yapmak için sürekli parçalı lineer ofset (continuous piece-wise linear offset, CPWLO) fonksiyonlarının kullanımını açıkladık.

\section{VLBI Gecikme Modeli}

Basit anlamda VLBI ölçü denklemi (gecikme modeli)

$-c . \tau=\boldsymbol{b}^{\oplus} . \boldsymbol{k}^{\odot}+\Delta \tau_{\text {saat }}+\Delta \tau_{\text {trop }}+\Delta \tau_{\text {iyono }}+\Delta \tau_{\text {relativistik }}$

biçimindedir. Burada, $c$ 1şığın (radyo dalgasının) vakum ortamdaki hızını, $\boldsymbol{k}^{\odot}$ radyo kaynağının (kuazar) uzay-sabit, barisentrik, ekvatoral bir göksel koordinat sisteminde, örneğin: ICRF2, tanımlı birim vektörünü, $\boldsymbol{b}^{\oplus}$ Yer-sabit, Yer-merkezli (jeosentrik), ekvatoral bir Yersel koordinat sisteminde, örneğin VTRF08, tanımlı VLBI antenlerinin baz vektörünü, $\Delta \tau_{\text {saat }}$ sabitlenmiş (hatasız kabul edilmiş) bir saate göre bağıl anlamda VLBI istasyonlarındaki saatlerin senkronizasyon ve frekans tutarsızlıklarından kaynaklı saat hatalarından ötürü oluşan sinyal gecikmesini, $\Delta \tau_{\text {trop }}$ troposfer sinyal gecikmesini, $\Delta \tau_{\text {iyono }}$ iyonosfer sinyal gecikmesini ve $\Delta \tau_{\text {relativistik }}$ relativistik etkiler sonucu oluşan sinyal gecikmesini göstermektedir.

$\mathrm{Bu}$ makalede açıklanan VLBI gecikme modeli temel olarak yeryüzünden galaksimiz dışındaki uzay objelerine gerçekleştirilen ölçülerin analizleri için tasarlanmıştır. VLBI uzay jeodezisi tekniğinin ölçülen büyüklükleri, istasyonlardaki atomik saatlerden okunan zaman değerleri ile etiketlenmiş radyo dalgası sinyalleridir. VLBI saatleri Eşgüdümlü Evrensel Zaman (Universal Time Coordinated, UTC) atomik zaman sistemine (ölçeğine) senkronize edilmişlerdir. ICRF kataloğu kuazar (radyo kaynağı) koordinatları Güneş Sistemi Merkezli Göksel Referans Sisteminde (Barycentric Celestial Reference System, BCRS) (uzay-sabit, orijin: güneş sisteminin ağırlık merkezi, ekvatoral) tanımlıdır. VLBI antenlerinin koordinatları Uluslararası Yersel Referans Sisteminde (International Terrestrial Reference System, ITRS) (Yer-sabit, orijin: Yer kitlesinin ağırlık merkezi, ekvatoral) tanımlıdır. VLBI ölçü denklemlerinin oluşturulmasında kullanılan bir diğer koordinat sistemi ise Yer-Merkezli 
Göksel Referans Sistemi (Geocentric Celestial Reference System, GCRS) BCRS eksen sistemine göre kinematik dönüklüğü olmayan orijini Yer'in ağırlık merkezinde olan bir referans sistemidir. Hesaplanan gecikme (ölçülerin yaklaşık değerleri) GCRS'de tanımlı olmak üzere 2 numaralı VLBI istasyonuna sinyalin varış anından, $t_{2}, 1$ numaralı istasyona aynı sinyalin varış anı, $t_{1}$ farkıdır (Petit ve Luzum 2010). En az iki veya daha fazla VLBI istasyonunun bir kuazara yaptığ gözlem sonucu en az bir veya daha fazla gecikme ölçüsünün elde edilmesi işlemine tarama (scan) adı verilir. Taramaların ilk istasyonlarındaki saat okumasından elde edilen UTC (Universal Time Coordinated) zaman etiketi, $t_{1}$ yaklaşı 1 değerlerin hesaplanmasına konu olan tüm skaler ve vektörel büyüklüklerin (örneğin: troposfer hidrostatik (kuru) sinyal gecikmesi, anten TRF koordinatlarına getirilen gelgitsel düzeltmeler, Yer'in ağırlık merkezinin barisentrik hız vektörü) zaman referansı (epoğu) olarak ele alınır. Bununla birlikte, UTC zaman ölçeğinde tanımlı $t_{1}$, hesabı gerçekleştirilecek büyüklüğün tanımlı olduğu zaman ölçeklerine dönüştürüldükten sonra kullanılmalıdır: Örneğin, Yersel zaman (terrestrial time, TT), temel (standart) referans epoğundan (J2000: 1 Ocak 2000, 12 UTC) itibaren julyen yüzyıllarında Yersel zaman. İlk olarak taramanın gerçekleştirildiği andaki, $t_{1}$ anten TRF koordinatları hız düzeltmeleri, eksentrisiteler, gel-git ve yükleme etkileri (katı Yer gel-gitleri, okyanus gel-gitsel yüklemeleri, gel-gitsel ve gel-gitsel olmayan atmosfer yüklemeleri, kutup gel-gitleri) VLBI antenlerinin TRF katalog koordinatlarından indirgenir. Böylece, taramanın gerçekleştirildiği andaki anten TRF koordinatları $\left(\boldsymbol{x}_{\text {ITRS }}\right)$ elde edilir. Sonrasında, ölçü (tarama) epoğundaki ITRS ve GCRS arasındaki dönüklük dönüşümü

$$
\boldsymbol{x}_{G C R S}=\boldsymbol{Q}(t) \cdot \boldsymbol{R}(t) \cdot \boldsymbol{W}(t) \cdot \boldsymbol{x}_{I T R S}
$$

eşitliğinden elde edilir. Burada $\boldsymbol{Q}(t), \boldsymbol{R}(t)$ ve $\boldsymbol{W}(t)$ sırası ile CIP'nin GCRS'deki hareketinden (nutasyon) kaynaklanan, ITRS'in GCRS'e göre üçüncü eksen dönüklüğünden kaynaklanan ve CIP'nin ITRS'deki hareketinden (kutup gezinmesi) kaynaklanan dönüklük matrisleridir. (2) eşitliğinde verilen $t$, tarama (ölçü) epoğu, $t_{1}$ 'in Yersel zaman (TT) ölçeğinde julyen yüzyılları biriminde temel (standart) referans epoğundan, J2000 (1 Ocak 2000, 12 UTC) itibaren karş1lı̆̆ıdır:

$t=(T T-51544.5) / 36525$

$T T=U T C+(T T-T A I)-(U T C-T A I)$,

$T T=t_{1}+(32.184-$ slçrama saniyeleri $) / 86400$.

(4) eşitliğinde verilen TAI, uluslararası atomik zaman (international atomic time), TT - TAI konvansiyon gereği 32.184 saniye ve UTC - TAI, sıçrama saniyeleri'dir (leap seconds). IERS tarafindan en son duyurulan 1 Ocak 2009 ve 1 Temmuz 2012 tarihleri arasında geçerli olan slçrama saniyeleri $=U T C-T A I=-34$ 'tür.

"Ekinoks'u temel alan" (equinox based) veya "Göksel Ortalama Orijin'i (Celestial Intermediate Origin (CIO)) temel alan" dönüşüm konvansiyonlarından bağımsız olarak kutup gezinmesinden kaynaklanan dönüşüm matrisi, diğer bir ifade ile ITRS ve Yersel Ortalama Referans Sistemi (Terrestrial Intermediate Reference System, TIRS) arasındaki kinematik ilişki,
$\boldsymbol{W}(t)=\boldsymbol{R}_{3}\left(s^{\prime}\right) \cdot \boldsymbol{R}_{2}\left(x_{p}\right) \cdot \boldsymbol{R}_{1}\left(y_{p}\right)$

eşitliğinden elde edilir. Burada $x_{p}$ ve $y_{p}$ CIP'nin ITRS'de tanımlı "kutup koordinatlarıdır". CIP kutup koordinatlarının; $x_{p}$ Greenwich meridyeni doğrultusundaki, $y_{p}$ ise $270^{\circ}$ doğu boylamı doğrultusundaki bileşenleridir. $\boldsymbol{R}_{1}, \boldsymbol{R}_{2}$ ve $\boldsymbol{R}_{3}$ herhangi bir kartezyen koordinat sisteminde birinci, ikinci ve üçüncü eksen dönüklük matrisleridir. s' büyüklüğü (dönüklüğü, açısı) "Yersel Ortalama Orijin Konumlandirıcısı (Terrestrial Intermediate Origin (TIO) locater)" olarak adlandırılır. TIO konumlandırıcısı $\left(s^{\prime}\right)$, "non-rotating origin (NRO)" konvansiyonunun kinematik tanımı gereği, ITRS'de tanımlı $\mathrm{t}$ epoğundaki CIP'nin belirlediği ekvator üzerindeki TIO'nun konumudur. $s^{\prime}$, kutup gezinmesindeki çok büyük değişikliklere duyarlıdır. $\mathrm{Bu}$ nedenle, Chandlerian ve yıllık (annual) kutup gezinmeleri periyotlarının ortalama genliklerinden faydalanılarak TIO konumlandırıcısı $\left(s^{\prime}\right)$ (Capitaine vd. 1986; Lambert ve Bizouard 2002):

$s^{\prime}=-47 \cdot 10^{-6} \cdot t$.

eşitliğinden elde edilir.

"Göksel Ortalama Orijin'i temel alan (CIO based)" dönüşüm konvansiyonuna göre: Yer'in CIP ekseni etrafindaki dönüklük matrisi, diğer bir ifade ile Yersel Ortalama Referans Sistemi, TIRS ve Göksel Ortalama Referans Sistemi (Celestial Intermediate Reference System, CIRS) arasındaki üçüncü eksen dönüklüğü,

$\boldsymbol{R}(t)=\boldsymbol{R}_{3}\left(-E R A\left(t_{u}\right)\right)$

eşitliğinden elde edilir. (7) eşitliğinde ERA Yer dönüklük açısı (Earth rotation angle), $t$ epoğundaki CIP'nin belirlediği ekvator üzerindeki CIO ve TIO arasındaki ekvator boyunca olan açıdır. ERA, kutup gezinmesinden kaynaklanan düzeltilme getirilmiş Evrensel Zaman, UT1 kullanılarak aşağıdaki eşlitlikten elde edilir:

$E R A\left(t_{u}\right)=2 \pi\left(E R A_{0}+a k \cdot t_{u}\right)$

(8) eşitliğinde $a k=1.00273781191135448, \quad E R A_{0}=$ 0.7790572732640 ve $t_{u}$ temel referans epoğundan (J2000) itibaren gün zaman biriminde ilgili ölçünün epoğudur $\left(t_{u}=U T 1-51544.5\right)$. Ölçü epoğu, $t_{1}$ 'in (UTC zaman ölçeğinde, MJD (modified julian date) zaman biriminde) UT1 zaman ölçeğindeki karşılığı

$U T 1=t_{1}+\Delta U T 1 / 86400$

eşitliğinden elde edilir. (9) eşitliğinde verilen 86400, zaman saniyesi birimindeki $\Delta U T 1$ değerini gün zaman birimine dönüştürür.

CIP'nin GCRS'deki hareketinden kaynaklanan “Göksel Ortalama Orijin'i (Celestial Intermediate Origin, CIO)" temel alan dönüşüm matrisi, diğer bir ifade ile CIRS ve GCRS arasındaki kinematik ilişki,

$\boldsymbol{Q}(t)=\boldsymbol{R}_{3}(-E) \cdot \boldsymbol{R}_{2}(-d) \cdot \boldsymbol{R}_{3}(E) \cdot \boldsymbol{R}_{3}(s)$

eşitliğinden elde edilir. Burada $E, d$, ve $s$ büyüklükleri CIP'nin GCRS'deki birim kartezyen koordinatlarından

$E=\arctan \frac{Y}{X}$, 
$d=\arccos Z=\arccos \left(\sqrt{1-X^{2}+Y^{2}}\right)$,

$s=s_{0}-\frac{X Y}{2}$.

eşitlikleri ile hesaplanır. (10) eşitliğindeki, s büyüklüğü “CIO konumlandırıcısı (CIO locater)" olarak adlandırılır. CIP, GCRS'de presesyon ve nutasyon hareketi yapar. Dolayısı ile her epok için CIP'nin GCRS'deki konumu değişir. CIO konumlandirıcisı $(s)$, "non-rotating origin (NRO)" konvansiyonunun kinematik tanımı gereği, GCRS'de tanımlı $t$ epoğundaki CIP'nin belirlediği ekvator üzerindeki CIO'nun konumudur (Capitaine vd. 2000, 2002; Petit ve Luzum 2010). $t_{1}$ ölçü epoğundaki VLBI antenlerinin (2) eşitliğinde verilen $\boldsymbol{x}_{G C R S}$ koordinatları, $\boldsymbol{x}_{i}^{\oplus}\left(t_{1}\right)$ elde edildikten sonra gravitasyonel, vakum ve geometrik sinyal gecikmeleri hesaplanır. Gravitasyonel, vakum ve geometrik sinyal gecikmelerine ilişkin modeller 2.1 ve 2.2 alt bölümlerinde detaylı bir biçimde açıklanmıştır.

\subsection{Gravitasyonel Gecikme}

Galaksi dışındaki radyo kaynağından gelen sinyal üzerinde $J$. gravite oluşturan uzay objesinin gravitasyonel geciktirmesi

$$
\Delta T_{\text {grav } J}=2 \frac{G M_{J}}{c^{3}} \ln \frac{\left|\boldsymbol{r}_{1 J}\right|+\boldsymbol{k}^{\odot} \cdot \boldsymbol{r}_{1 J}}{\left|\boldsymbol{r}_{2 J}\right|+\boldsymbol{k}^{\odot} \cdot \boldsymbol{r}_{2 J}},
$$

eşitliğinden hesaplanır. Burada $G$ Newton'un gravitasyonel sabiti, $M_{j} J$. gravite oluşturan uzay objesinin kitlesi, $c$ vakum ortamda 1şık hızı, $\boldsymbol{r}_{i J} J$. gravite oluşturan uzay objesinden $i$. antene (alıcıya) giden vektör, mutlak operatörü vektörün normunu, örneğin $|\boldsymbol{x}|=\sqrt{\sum x_{i}^{2}}$, göstermektedir. $\boldsymbol{k}^{\odot}$ barisentırdan (güneş sisteminin ağırlık merkezi) radyo kaynağına gravitasyonel ve aberasyon bükülmesinden bağımsız birim vektör

$$
\boldsymbol{k}^{\odot}=\left[\begin{array}{lll}
\cos \delta \cos \alpha & \cos \delta \sin \alpha & \sin \delta
\end{array}\right],
$$

biçimindedir. (15) eşitliğinde $\delta$ ve $\alpha$ kuazarın (radyo kaynağının) sırası ile BCRS'de tanımlı deklinasyon ve rektezansiyon koordinatlarıdır. Yer'in sinyal üzerinde oluşturduğu gravitasyonel gecikme miktarı

$$
\Delta T_{\text {grav } \oplus}=2 \frac{G M_{\oplus}}{c^{3}} \ln \frac{\left|\boldsymbol{x}_{1}^{\oplus}\right|+\boldsymbol{k}^{\odot} \cdot \boldsymbol{x}_{1}^{\oplus}}{\left|\boldsymbol{x}_{2}^{\oplus}\right|+\boldsymbol{k}^{\odot} \cdot \boldsymbol{x}_{2}^{\oplus}},
$$

eşitliğinden elde edilir. Burada $\boldsymbol{x}_{i}^{\oplus}, i$. antenin $t_{1}$ epoğundaki GCRS koordinat vektörünü ve $M_{\oplus}$ Yer'in kitlesini göstermektedir. $\boldsymbol{r}_{i J}$ vektörü, sinyalin $J$. gravite oluşturan uzay objesine en yakın geçtiği andaki epokda $\left(t_{i J}\right)$ hesaplanmalıdır. Bu nedenle $t_{1}$ (ölçü) epoğuna zaman düzeltmesi getirilerek, radyo dalgasının gravite oluşturan kitleye en yakın olduğu an, $t_{1 J}$ epoğu hesaplanır. $t_{1 J}$ anındaki $J$. gravite oluşturan uzay objesinin barisentrik konum vektörü, $\boldsymbol{x}_{J}^{\odot}$ ve $t_{1 J}$ epoğu, $t_{1 J}=t_{1}-\frac{\boldsymbol{k}^{\odot} \cdot\left(\boldsymbol{x}_{J}^{\odot}\left(t_{1}\right)-\boldsymbol{x}_{1}^{\odot}\left(t_{1}\right)\right)}{c}$

$\boldsymbol{x}_{J}^{\odot}\left(t_{1 J}\right)=\boldsymbol{x}_{J}^{\odot}\left(t_{1}\right)+\boldsymbol{v}_{J}^{\odot}\left(t_{1 J}-t_{1}\right)$

eşitliklerinden iteratif olarak hesaplanır. (17) eşitliklerinde verilen $\boldsymbol{x}_{J}^{\odot}$ ve $\boldsymbol{v}_{J}^{\odot}, J$. gravite oluşturan uzay objesinin sırası ile barisentrik konum ve hız vektörleridir. $J$. gravite oluşturan uzay objesi ve birinci anten arasındaki konum fark vektörü

$\boldsymbol{r}_{1 J}\left(t_{1}\right)=\boldsymbol{x}_{1}^{\odot}\left(t_{1}\right)-\boldsymbol{x}_{J}^{\odot}\left(t_{1 J}\right)$

eşitliğinden elde edilir. Eşitlik (18)'deki $i$. antenin $t_{1}$ epoğun-

daki $\boldsymbol{x}_{i}^{\odot}$ barisentrik koordinatları: Yermerkezinin barisentrik konum vektörü, $\boldsymbol{x}_{\oplus}^{\odot}\left(t_{1}\right)$ ve $i$. antenin GCRS koordinatları, $\boldsymbol{x}_{i}^{\oplus}\left(t_{1}\right)$ ile

$\boldsymbol{x}_{i}^{\odot}\left(t_{1}\right)=\boldsymbol{x}_{\oplus}^{\odot}\left(t_{1}\right)+\boldsymbol{x}_{i}^{\oplus}\left(t_{1}\right)$

eşitliğinden hesaplanır. $J$. gravite oluşturan uzay objesi ve ikinci anten arasındaki konum fark vektörü, $\boldsymbol{r}_{2 J}$ sinyalin birinci ve ikinci anten arasındaki gecikmesi sırasında ikinci antenin BCRS' de yer değiştirmesi dikkate alınarak,

$\boldsymbol{r}_{2 J}=\boldsymbol{x}_{2}^{\odot}\left(t_{1}\right)-\frac{\boldsymbol{v}_{\oplus}^{\odot}}{c}\left(\boldsymbol{k}^{\odot} \cdot \boldsymbol{b}^{\oplus}\right)-\boldsymbol{x}_{J}^{\odot}\left(t_{1 J}\right)$

eşitliğinden bulunur. Burada $\boldsymbol{v}_{\oplus}^{\odot}$, Yermerkezinin barisentrik hız vektörü ve $\boldsymbol{b}^{\oplus}, t_{1}$ epoğunda GCRS'de tanımlı antenlerin baz vektörünü, $\boldsymbol{x}_{2}^{\oplus}\left(t_{1}\right)-\boldsymbol{x}_{1}^{\oplus}\left(t_{1}\right)$, göstermektedir. Sinyal üzerindeki toplam gravitasyonel gecikme, Yer'i de içeren tüm gravite oluşturan uzay objelerinin oluşturduğu gecikmelerin skaler toplamidir:

$\Delta T_{\text {grav }}=\sum_{j} \Delta T_{\text {grav }}$

\subsection{Vakum Gecikme ve Geometrik Gecikme}

Toplam Yermerkezli vakum sinyal gecikmesi

$$
t_{v 2}-t_{v 1}=\frac{\Delta T_{\text {grav }}-\frac{\boldsymbol{k}^{\odot} \cdot \boldsymbol{b}^{\oplus}}{c}\left[1-\frac{(1+\gamma) \cdot U}{c^{2}}-\frac{\left|\boldsymbol{v}_{\oplus}^{\odot}\right|^{2}}{2 c^{2}}-\frac{\boldsymbol{v}_{\oplus}^{\odot} \cdot \omega_{2}^{\oplus}}{c^{2}}\right]-\frac{\boldsymbol{v}_{\oplus}^{\odot} \cdot \boldsymbol{b}^{\oplus}}{c^{2}}\left(1+\frac{\boldsymbol{k}^{\odot} \cdot \boldsymbol{v}_{\oplus}^{\odot}}{2 c}\right)}{1+\frac{\boldsymbol{k}^{\odot} \cdot\left(\boldsymbol{v}_{\oplus}^{\odot}+\omega_{2}^{\oplus}\right)}{c}}
$$

eşitliğinden elde edilir. Burada, BCRS için genel görelilik (general relativity) teorisine göre Lorentz katsayısı, $\gamma=1$ alınır. $\omega_{i}^{\oplus}, i$. antenin Yermerkezli hız vektörü ve $U$ Yer'in kitlesi ihmal edilerek ve yalnızca güneşin kitlesinden ötürü oluşan Yer'in ağırlık merkezindeki gravite potansiyeli:

$U=\frac{G M_{\odot}}{\left|\boldsymbol{r}_{\odot \oplus}\right|}$,

eşitliğinden hesaplanır. (23) eşitliğinde $\boldsymbol{r}_{\odot \oplus}$ güneşten Yer'in merkezine olan BCRS'de tanımlı vektör ve $M_{\odot}$ güneşin kitlesidir. (22) eşitliğinde, $t_{v i} i$. VLBI antenine radyo sinyali ulaştığ fer gecikmesi ve atmosfer bükülmesini içermeyen "vakum" Yermerkezli koordinat zamanı (geocentric coordinate time, $T C G)$ dır. Gravitasyonel ve vakum sinyal gecikmelerinin 
yaklaşık değerlerinin hesaplarında kullanılan bazı vektörel büyüklükler, örneğin: $\boldsymbol{x}_{\oplus}^{\odot}, \boldsymbol{v}_{\oplus}^{\odot}, \boldsymbol{x}_{J}^{\odot}, \boldsymbol{v}_{J}^{\odot}$ gezegensel almanaklardan, örneğin: JPL421, elde edilirler.

Geometrik sinyal gecikmesi, troposfer gecikmesinin vakum sinyal gecikmesine eklenmesi ile

$$
t_{g 2}-t_{g 1}=t_{v 2}-t_{v 1}+\delta t_{a t m 1} \frac{\boldsymbol{k}^{\odot} \cdot\left(\omega_{2}^{\oplus}-\omega_{1}^{\oplus}\right)}{c}
$$

elde edilir. Eşitlik (24)'de verilen $\delta_{a t m 1}, t_{1}$ ölçü epoğunda birinci antende ölçü doğrultusunda hidrostatik troposfer sinyal gecikmesi olarak düsünülebilir ve

$$
\delta_{\text {atm } 1}=\frac{\Delta L_{h}^{s t(1)}\left(t_{1}\right) \cdot m_{h}^{s t(1)}\left(\varepsilon, t_{1}\right)}{c},
$$

eşitliği ile hesaplanabilir. (25) eşitliğinde verilen $\Delta L_{h}^{s t(1)}\left(t_{1}\right), t_{1}$ ölçü epoğunda birinci antendeki troposfer zenit hidrostatik gecikmesi ve $\varepsilon$, ölçünün lokal yükselim açısıdır.

VLBI antenlerinin oluşturduğu baz vektörüne uygulanan kuazardan antenlere ulaşan sinyalin azimut ve yükselim açılarına bağımlı düzeltmeler termal deformasyonlar: $\tau_{\text {termal deft } 12}$, ofset düzeltmeleri: $\tau_{\text {eksen ofsetleri12 }}$ ve hidrostatik troposfer gecikmeleri: $\tau_{\text {trop } 12}$ geometrik sinyal gecikmesine eklenerek, toplam gecikme, $t_{2}-t_{1}$ :

$t_{2}-t_{1}=t_{g 2}-t_{g 1}+\tau_{\text {termal deft } 12}+\tau_{\text {eksen ofsetleri } 12}+\tau_{\text {trop } 12}$

şeklinde elde edilir. Ölçünün zenit uzaklığ $\left(90^{\circ}-\varepsilon\right)$ ve azimut açısı (ölçüye jeodezik kuzeyden saat ibresi yönünde olan yatay açı) aberasyon bükülmesini içerir. Böylece, $i$ anteni için Yermerkezinden (ağırlık merkezi) kuazara olan birim vektör, $\boldsymbol{k}_{i}^{\oplus}$

$\boldsymbol{k}_{i}^{\oplus}=\boldsymbol{k}^{\odot}+\frac{\boldsymbol{v}_{\oplus}^{\odot}+\omega_{i}^{\oplus}}{c}-\boldsymbol{k}^{\odot} \cdot \frac{\boldsymbol{k}^{\odot} \cdot\left(\boldsymbol{v}_{\oplus}^{\odot}+\omega_{i}^{\oplus}\right)}{c}$.

eşitliğinden hesaplanır. VLBI ölçülerinden iyonosfer sinyal gecikmeleri çift frekanslı ölçülerin lineer kombinasyonları alınarak indirgenir. Böylece parametre kestirimine konu olan indirgenmiş ölçü değerleri (observed minus computed, oc) her bir ölçü için:

$o c=\tau_{\text {ölçülen }}-\left(t_{2}-t_{1}\right)$.

şeklinde elde edilir. oc vektörü, senkronizasyon uyuşumsuzluğu ve frekans tutarsızlıklarından kaynaklanan saat hatalarını, troposfer 1slak gecikmelerini, troposferin azimutsal asimetrik gecikmelerini ve $\left(t_{2}-t_{1}\right)$ yaklaşı (öncül) gecikme değerlerinin hesaplanmasında kullanılan modellerdeki değerlerin eksikliklerini (örneğin: kestirilen (predicted) Yer dönme parametreleri, EOP hatalarını) içerir ve bunlar yaklaşık değerlerin düzeltmeleri şeklinde parametre kestirimi sonucu duyarlı olarak kestirilir (Petit ve Luzum 2010; Böhm vd. 2011a; Teke 2011).

\subsection{Saat Senkronizasyon ve Frekans Tutarsızlıklarının Oluşturduğu Gecikme}

VLBI analizinde Yeryüzüne sabitlenmiş iki adet saatin (çoğunlukla hydrogen maser) arasındaki saat hataları, kuadratik bir polinoma ek olarak sürekli parçalı lineer ofsetler (continuous piecewise linear offsets (CPWLO)) fonksiyonu ile en küçük kareler (EKK) kestirim yönteminden
$\Delta \tau_{\text {saat }}(t)=\Delta t_{\text {saat }}^{\text {poly }}(t)+\Delta t_{\text {saat }}^{\text {cpwlo }}(t)$

belirlenebilir (Teke 2011). Her saat için oluşturulan polinom

$\Delta \tau_{\text {saat }}^{\text {poly }}(t)=\beta_{0}+\beta_{1}\left(t-t_{0}\right)+\beta_{2}\left(t-t_{0}\right)^{2}$

şeklindedir. Burada $t, U T C$ ölçü epoğu, $t_{0}$ keyfi seçilen bir epok, örneğin oturumun başladığı zaman, $\beta_{j}(j=0,1,2)$ polinomun katsayılarıdır. Sürekli parçalı lineer ofsetler (CPWLO) fonksiyonu ile gün-içi zamansal çözünürlükte parametre kestrimi (örneğin, saat hatalarından kaynaklanan sinyal gecikmesi, $\left.\Delta t_{\text {saat }}^{\text {cpwlo }}(t)\right)$ Bölüm 3'de detaylı olarak açıklanmaktadır.

\subsection{Troposfer Gecikmesi}

Troposfer (atmosferin elektriksel nötr bölümü), radyo kaynağından alıcıya (antene) elektromanyetik radyo dalgasının yayılması esnasında troposfer ışın yolu boyunca gecikmesine ve bükülmesine neden olur. Troposferin sinyal yolunda (s) oluşan (eğik, slant) gecikme $\left(\Delta L, \Delta \tau_{\text {trop }}\right.$ notasyonunun metre birimindeki karşılığı: $\Delta L=\mathrm{c} \cdot \Delta \tau_{\text {trop }}$ ) alıcıdan troposferin üst sinırına $\left(H_{\text {trop }}\right)$ kadar sinyal yolu boyunca hidrostatik (kuru) ve sslak refraktivite değerlerinin $\left(N_{h, w}\right)$ integrali (refraktif indeks, $n$ ) ile elde edilir:

$$
\begin{aligned}
\Delta L & =\int_{s}(n-1) d s=10^{-6} \int_{s} N d s \\
& =10^{-6} \int_{0}^{H_{\text {trop }}}\left[N_{h}(s)+N_{w}(s)\right] d s
\end{aligned} .
$$

(31) eşitliği yükselim açısı bağımlı kısım (küresel kısım) ve troposfer grandyanları olarak da bilinen azimutsal asimetrik kısım (küresel-olmayan kısım) olmak üzere iki bölüme ayrıştırılabilir (Davis vd. 1993):

$$
\Delta L=\Delta L_{\text {küresel }}(\varepsilon)+\Delta L_{\text {küresel-olmayan }}(\alpha, \varepsilon) .
$$

Yükselim açısına bağımlı kısım (Davis vd. 1993)

$$
\Delta L_{\text {küresel }}(\varepsilon)=Z H D m_{h}(\varepsilon)+Z W D m_{w}(\varepsilon) .
$$

şeklinde formulüze edilebilir. (33) eşitliğinde verilen, $\varepsilon$ ölçünün lokal yataydan yükselim açısı ve $Z H D$ zenit hidrostatik gecikmesi ki istasyon noktasında yapılacak toplam basınç ölçüsü, $p$ ve istasyon noktasının koordinatları (enlem, $\varphi$ ve yükseklik, $h$ ) ile aşağıdaki eşitlikten hesaplanabilir (Saastamoinen 1972):

$$
Z H D=0.0022768 \frac{p}{\left(1-0.00266 \cdot \cos (2 \varphi)-0.28 \cdot 10^{-6} \cdot h\right)}(34)
$$

(33) eşitliğinde; $Z W D$ zenit 1slak gecikmesi, $m_{h}(\varepsilon)$ ve $m_{w}(\varepsilon)$ hidrostatik ve islak troposfer izdüşüm fonksiyonlarıdır (örneğin; Niell 1996; Böhm vd. 2006). Troposfer izdüşüm fonksiyonları aşağıdaki şekilde ifade edilirler (Marini 1972): 


$$
m_{h, w}(\varepsilon)=\frac{1+\frac{a_{h, w}}{1+\frac{b_{h, w}}{1+c_{h, w}}}}{\sin (\varepsilon)+\frac{a_{h, w}}{\sin (\varepsilon)+\frac{b_{h, w}}{\sin (\varepsilon)+c_{h, w}}}} .
$$

Burada $a_{h, w}, b_{h, w}$ ve $c_{h, w}$ troposfer izdüşüm fonksiyonunun hidrostatik ve 1slak katsayılarıdır. Tüm troposfer izdüşüm fonksiyonları bu katsayıların belirlenmesi yönü ile birbirlerinden ayrışırlar. (32) eşitliğinde verilen troposferin azimutsal asimetrik kısmı (küresel-olmayan kısmı) aşağıdaki eşitlikle modellenebilir (Davis vd. 1993):

$$
\Delta L_{\text {kïresel olmayan }}(\alpha, \varepsilon)=m_{g}(\varepsilon)\left[G_{n} \cos (\alpha)+G_{e} \sin (\alpha)\right]
$$

(36) eșitliğinde $\alpha$ azimut (ölçüye jeodezik kuzeyden saat ibresi yönünde olan yatay açı), $G_{n}$ ve $G_{e}$ kuzey ve doğu troposfer toplam (1slak artı hidrostatik) yatay gradyanları ve $m_{\mathrm{g}}(\varepsilon)$ gradyan izdüşüm fonksiyonudur. MacMillan (1995)'e göre gradyan izdüşüm fonksiyonu,

$m_{g}(\varepsilon)=m_{h}(\varepsilon) \cot (\varepsilon)$

Chen ve Herring (1997)'ye göre gradyan izdüşüm fonksiyonu ise

$$
m_{g}(\varepsilon)=\frac{1}{\tan (\varepsilon) \sin (\varepsilon)+C}
$$

şeklindedir. Burada toplam (1slak artı hidrostatik) gradyanların kestirimi için, $C=0.0032$ alınır (Herring 1992). Ĕger (38) eşitliğinde $C=0.0007$ alınırsa 1slak gradyanlar için izdüşüm fonksiyonu değerleri elde edilir ve (37) eşitliğinde verilen MacMillan'ın (1995) modeline çok yakın değerler elde edilir (Böhm vd. 2011b).

$\mathrm{Bu}$ bölümde açıklanan troposfer gecikme modeli, VLBI, Global Navigation and Satellite Systems (GNSS) ve Doppler Orbitography and Radio Positioning Integrated by Satellite (DORIS) gibi radyo dalgası kullanan uzay jeodezisi tekniklerinin ölçülerinin analizinde kullanılır. Çoğu VLBI oturumunun analizinde ilk olarak ZHD (34) eşitliğinden elde edilir, sonra herhangi bir hidrostatik izdüşüm fonksiyonu ile, örneğin $V M F 1_{h}$ (Böhm vd. 2006), ilgili yükselim açısındaki ölçüye izdüşürülür ve ardından parametre kestirimi öncesi hesaplanan bu hidrostatik gecikme değerleri ölçülerden indirgenir. VLBI ölçülerinden kestirimi yapılan troposfer parametreleri; $Z W D, G_{n}$ ve $G_{e}$ 'dir (Teke vd. 2009).

Troposfer sslak gecikmeleri mekansal ve zamansal anlamda çok değişken olan sslak refraktivite sebebiyle tam modellenememekte ve bunun sonucu olarak da uzay jeodezisi tekniklerinin tümünde troposfer ölçülerdeki en büyük hata kaynağı olma pozisyonunu korumaktadır. Troposfer kaynaklı modellenemeyen ölçü hataları kestrimi yapılan diğer parametrelere dağılmakta (troposfer gecikme parametreleri ile diğer parametreler arasındaki korelasyonlar oranında) ve özellikle anten TRF koordinatlarının radyal (yükseklik) bileşenine yansımaktadırlar (Herring 1986; Davis vd. 1991). Uzay jeodezisi ölçülerindeki troposfer kaynaklı hataların enleme bağlı ve mevsimsel deği- şimler gösterdiği radyo dalgası kullanan uzay jeodezisi teknikleri; VLBI, GPS ve DORIS ölçülerinin analizinden elde edilen troposfer kestirimlerinin karşılaştırılması sonucu ortaya çıkarılmıştır (Teke vd. 2011).

\subsection{VLBI Gecikme Modelinin Yer Dönme Parametrelerine, Anten ve Kuazar Koordinatlarına Göre Kısmi Türevleri}

VLBI gecikme modelinin kestirimi yapılacak bir parametreye göre kısmi türevi, tüm diğer parametreler sabit tutulup kısmi türevi alınan parametre değiştirildiğinde ilgili ölçünün ne kadar değişeceğini diğer bir ifade ile gecikme modelinin kısmi türevi alınan parametreye olan duyarlığını gösterir. Anlatım kolaylığı açısından, kestirim parametresine göre kısmi türevi alınan VLBI gecikme modeli olarak basit bir gecikme modeli seçilmiştir. Bu nedenle, kısmi türevlerin alınmasında relativistik etki modelleri dikkate alınmamıştır. Böylece, gravitasyonel gecikme modeli ve istasyon konumlarının GCRS'den BCRS'e dönüşümü (Lorentz dönüşümü), güneş sisteminin ve Yer'in kitlelerinin ağırlık merkezleri (koordinat sistemlerinin orijinleri) farklılıklarından dolayı gerçekleştirilen dönüşüm, dikkate alınmamıştır. Kısmi türevlerin alınmasında VLBI gecikme modeli olarak aşağıdaki eşitlik kullanılabilir:

$$
\tau=\boldsymbol{k}^{\odot} \cdot \boldsymbol{Q} \cdot \boldsymbol{R} \cdot \boldsymbol{W} \cdot \boldsymbol{b}^{\oplus}
$$

(39) eşitliğinde, $\boldsymbol{k}^{\odot}$ BCRS'de tanımlı ve $\boldsymbol{b}^{\oplus}$ TRF'de tanımlıdır. (39) eşitliğinde verilen VLBI gecikme modelinin CIP'nin TRF'de tanımlı Greenwich meridyeni dogrultusundaki koordinat bileşenine, $x_{p}$ göre kısmi türevi:

$$
\frac{\partial \tau}{\partial x_{p}}=\boldsymbol{k}^{\odot} \cdot \boldsymbol{Q} \cdot \boldsymbol{R} \cdot \frac{\partial \boldsymbol{W}}{\partial x_{p}} \cdot \boldsymbol{b}^{\oplus},
$$

burada

$$
\frac{\partial \boldsymbol{W}}{\partial x_{p}}=\boldsymbol{R}_{3}\left(s^{\prime}\right) \cdot\left[\begin{array}{ccc}
-\sin x_{p} & 0 & -\cos x_{p} \\
0 & 0 & 0 \\
\cos x_{p} & 0 & -\sin x_{p}
\end{array}\right] \cdot \boldsymbol{R}_{1}\left(y_{p}\right) .
$$

(39) eşitliğinde verilen VLBI gecikme modelinin CIP'nin TRF'de tanımlı $270^{\circ}$ doğu boylamı doğrultusundaki koordinat bileşenine, $y_{p}$ göre kısmi türevi:

$$
\frac{\partial \tau}{\partial y_{p}}=\boldsymbol{k}^{\odot} \cdot \boldsymbol{Q} \cdot \boldsymbol{R} \cdot \frac{\partial \boldsymbol{W}}{\partial y_{p}} \cdot \boldsymbol{b}^{\oplus}
$$

burada

$$
\frac{\partial \boldsymbol{W}}{\partial y_{p}}=\boldsymbol{R}_{3}\left(s^{\prime}\right) \cdot \boldsymbol{R}_{2}\left(x_{p}\right) \cdot\left[\begin{array}{ccc}
0 & 0 & 0 \\
0 & -\sin y_{p} & \cos y_{p} \\
0 & -\cos y_{p} & -\sin y_{p}
\end{array}\right]
$$

Gecikme modelinin Evrensel Zamana (UT1) göre kısmi türevi:

$$
\frac{\partial \tau}{\partial(U T 1)}=\boldsymbol{k}^{\odot} \cdot \boldsymbol{Q} \cdot \frac{\partial \boldsymbol{R}}{\partial(-E R A)} \cdot \frac{\partial(-E R A)}{\partial(U T 1)} \cdot \boldsymbol{W} \cdot \boldsymbol{b}^{\oplus},
$$


burada

$$
\frac{\partial \boldsymbol{R}}{\partial(-E R A)}=\left[\begin{array}{ccc}
-\sin (-E R A) & \cos (-E R A) & 0 \\
-\cos (-E R A) & -\sin (-E R A) & 0 \\
0 & 0 & 0
\end{array}\right],
$$

ve

$$
\frac{\partial(-E R A)}{\partial(U T 1)}=-a k=-1.00273781191135448
$$

Gök ortalama kutbunun (CIP) CRS'deki hareketinden ötürü dört adet dönüklük matrisinden oluşan (10) eşitliğinde verilen dönüşüm matrisinin elemanlarının; $E, d$ ve $s$ nasıl hesaplanacağ 1 (11, 12 ve 13$)$ eşitliklerinde verilmiştir. VLBI gecikme modelinin, CIP'nin CRF'de tanıml $X$ koordinat bileşenine (nutasyon $X$ ofseti) göre kısmi türevi:

$$
\frac{\partial \tau}{\partial X}=\boldsymbol{k}^{\odot} \cdot \frac{\partial \boldsymbol{Q}}{\partial X} \cdot \boldsymbol{R} \cdot \boldsymbol{W} \cdot \boldsymbol{b}^{\oplus},
$$

burada

$$
\begin{aligned}
\frac{\partial \boldsymbol{Q}}{\partial X}= & \frac{\partial \boldsymbol{R}_{3}(-E)}{\partial(-E)} \cdot \frac{\partial(-E)}{\partial X} \cdot \boldsymbol{R}_{2}(-d) \cdot \boldsymbol{R}_{3}(E) \cdot \boldsymbol{R}_{3}(s) \\
& +\boldsymbol{R}_{3}(-E) \cdot \frac{\partial \boldsymbol{R}_{2}(-d)}{\partial(-d)} \cdot \frac{\partial(-d)}{\partial X} \cdot \boldsymbol{R}_{3}(E) \cdot \boldsymbol{R}_{3}(s) \\
& +\boldsymbol{R}_{3}(-E) \cdot \boldsymbol{R}_{2}(-d) \cdot \frac{\partial \boldsymbol{R}_{3}(E)}{\partial E} \cdot \frac{\partial E}{\partial X} \cdot \boldsymbol{R}_{3}(s) \\
& +\boldsymbol{R}_{3}(-E) \cdot \boldsymbol{R}_{2}(-d) \cdot \boldsymbol{R}_{3}(E) \cdot \frac{\partial \boldsymbol{R}_{3}(s)}{\partial s} \cdot \frac{\partial s}{\partial X}
\end{aligned}
$$

(48) ve (53) eşitliklerindeki, $\boldsymbol{R}_{i}(i=1,2,3)$ herhangi bir kartezyen koordinat sisteminde birinci, ikinci ve üçüncü eksen dönüklük matrisleridir ve $\partial \boldsymbol{R}_{i} / \partial$ ise dönüklük matrislerinin ilgili dönüklük açılarına göre kısmi türevleridir. (48) eşitliğinde verilen ara değerler

$$
\begin{aligned}
& \frac{\partial(-E)}{\partial X}=\frac{Y}{X^{2}+Y^{2}}, \\
& \frac{\partial(-d)}{\partial X}=\frac{X}{\sqrt{1-\left(X^{2}+Y^{2}\right)} \sqrt{X^{2}+Y^{2}}}, \\
& \frac{\partial s}{\partial X}=\frac{-Y}{2}
\end{aligned}
$$

şeklinde elde edilir. VLBI gecikme modelinin, CIP'nin CRF'de tanımlı $Y$ koordinat bileşenine (nutasyon $Y$ ofseti) göre k1smi türevi:

$$
\frac{\partial \tau}{\partial Y}=\boldsymbol{k}^{\odot} \cdot \frac{\partial \boldsymbol{Q}}{\partial Y} \cdot \boldsymbol{R} \cdot \boldsymbol{W} \cdot \boldsymbol{b}^{\oplus},
$$

burada

$$
\begin{aligned}
\frac{\partial \boldsymbol{Q}}{\partial Y}= & \frac{\partial \boldsymbol{R}_{3}(-E)}{\partial(-E)} \cdot \frac{\partial(-E)}{\partial Y} \cdot \boldsymbol{R}_{2}(-d) \cdot \boldsymbol{R}_{3}(E) \cdot \boldsymbol{R}_{3}(s) \\
& +\boldsymbol{R}_{3}(-E) \cdot \frac{\partial \boldsymbol{R}_{2}(-d)}{\partial(-d)} \cdot \frac{\partial(-d)}{\partial Y} \cdot \boldsymbol{R}_{3}(E) \cdot \boldsymbol{R}_{3}(s) \\
& +\boldsymbol{R}_{3}(-E) \cdot \boldsymbol{R}_{2}(-d) \cdot \frac{\partial \boldsymbol{R}_{3}(E)}{\partial E} \cdot \frac{\partial E}{\partial Y} \cdot \boldsymbol{R}_{3}(s) \\
& +\boldsymbol{R}_{3}(-E) \cdot \boldsymbol{R}_{2}(-d) \cdot \boldsymbol{R}_{3}(E) \cdot \frac{\partial \boldsymbol{R}_{3}(s)}{\partial s} \cdot \frac{\partial s}{\partial Y}
\end{aligned}
$$

(53) eşitliğinde verilen ara değerler,

$\frac{\partial(-E)}{\partial Y}=\frac{-X}{X^{2}+Y^{2}}$,

$\frac{\partial(-d)}{\partial Y}=\frac{Y}{\sqrt{1-\left(X^{2}+Y^{2}\right)} \sqrt{X^{2}+Y^{2}}}$,

$\frac{\partial s}{\partial Y}=\frac{-X}{2}$

şeklinde elde edilir. VLBI gecikme modelinin, kuazarın BCRS'de tanımlı rektezansiyon koordinat bileşenine, $\alpha$ göre kısmi türevi:

$\frac{\partial \tau}{\partial \alpha}=\frac{\partial \boldsymbol{k}^{\odot}}{\partial \alpha} \cdot \boldsymbol{Q} \cdot \boldsymbol{R} \cdot \boldsymbol{W} \cdot \boldsymbol{b}^{\oplus}$,

burada

$\frac{\partial \boldsymbol{k}^{\odot}}{\partial \alpha}=\left[\begin{array}{lll}-\cos \delta \sin \alpha & \cos \delta \cos \alpha & 0\end{array}\right]$

Gecikme modelinin, kuazarın BCRS' de tanımlı deklinasyon koordinat bileşenine, $\delta$ göre kısmi türevi:

$\frac{\partial \tau}{\partial \delta}=\frac{\partial \boldsymbol{k}^{\odot}}{\partial \delta} \cdot \boldsymbol{Q} \cdot \boldsymbol{R} \cdot \boldsymbol{W} \cdot \boldsymbol{b}^{\oplus}$,

burada

$\frac{\partial \boldsymbol{k}^{\odot}}{\partial \delta}=\left[\begin{array}{lll}-\sin \delta \cos \alpha & -\sin \delta \sin \alpha & \cos \delta\end{array}\right]$.

VLBI gecikme modelinin, antenlerin TRF'de tanımlı konumuna göre, örneğin birinci antenin TRF'de tanımlı $X$ koordinat bileşenine göre kısmi türevi:

$\frac{\partial \tau}{\partial X_{s t 1}}=\boldsymbol{k}^{\odot} \cdot \boldsymbol{Q} \cdot \boldsymbol{R} \cdot \boldsymbol{W} \cdot \frac{\partial \boldsymbol{b}^{\oplus}}{\partial X_{s t 1}}$,

burada

$\frac{\partial \boldsymbol{b}^{\oplus}}{\partial X_{s t 1}}=\left[\begin{array}{l}1 \\ 0 \\ 0\end{array}\right]$.

Gecikme modelinin kestirim parametrelerine göre kısmi türevlerine örnek olarak; üç baz ve iki kuazar ile gerçekleştirilen 16 Agustos 2008 tarihli 24 saatlik bir VLBI oturumu için gecikme modelinin Evrensel Zamana, UT1 göre kısmi türevi (44) eşitliğinden hesaplanmıştır. Bu örnekte, Kokee (Hawaii, USA) - Wettzell (Almanya) bazı 10360 km uzunluğunda doğu-batı doğrultulu, Westford (USA) - Wettzell 
bazı da aynı șekilde doğu-batı doğrultulu ve 6000 km uzunluğunda, Onsala (Isvec) - Wettzell bazı ise kuzey-güney doğrultulu 920 km uzunluğundadır. Kokee ve Wettzell VLBI istasyonlarının 1357+769 kuazarına 9:00 ve 21:00 UTC'de gerçekleștirdikleri ölçüler dikkate alındığında $U T 1$ 'daki bir milisaniyelik (zaman saniyesi) değişim ilgili ölçülerde yaklaşık $15 \mathrm{~cm}$ 'lik bir gecikmeye (değişime) neden olmaktadır (kırmızı halkalar, Şekil 2). Bununla birlikte, 3:00 ve 15:00 $U T C$ 'de $U T 1$ 'daki değişim ilgili ölçülerde hemen hemen hiçbir gecikmeye (değişime) neden olmamaktadır. Çünkü, UT1 parametresi doğu-batı doğrultulu bazlarda (doğu-batı doğrultulu VLBI istasyonlarının yaptıkları ölçülerde) bazın uzayda gözlem (ölçü) yapılan kuazara göre dönüklügüne duyarlıdır. Diğer bir ifade ile kuazar ile bazı dik kesen bir epokda yapılan ölçünün duyarlığı UT1 duyarlığının yüksek olması için gereklidir sonucuna varılır.

\section{Gün-içi Zamansal Çözünürlükte Parametre Kestirimi için Sürekli Parçalı Lineer Ofset Fonksiyonları}

İlk epok için ofset ve diğer epoklarda ofset lineer değişimlerinin (rate) kestiriminin yapıldığı sürekli parçalı lineer (cotinuous piecewise linear, CPWL) fonksiyonlarına benzer fakat üstün olarak direk tüm epoklarda (UTC tam saatleri, bölümleri veya katlarında) ofsetlerin kestiriminin yapıldığ 1 sürekli parçalı lineer ofset (cotinuous piecewise linear offset, CPWLO) fonksiyonlarını,

$x_{i}=x_{1}+\frac{t-t_{1}}{t_{2}-t_{1}}\left(x_{2}-x_{1}\right)$

VLBI ölçülerinden gün-içi jeodezik parametrelerin kestiriminde ilk kez kullandık (Böhm vd. 2011a; Teke 2011). (63) eşitliğinde verilen $x_{1}$ ve $x_{2}$ : CPWLO UTC tam saatleri, bölümleri veya katları kestirim epoklarındaki $\left(t_{1}\right.$ ve $\left.t_{2}\right)$ kestirim parametrelerini, $t$ ölçü epoğunu, $x_{\mathrm{i}}$ ise $t$ ölçü epoğunda ilgili bilinmeyen parametrenin değerini göstermektedir. Sürekli parçalı lineer ofsetlerin (CPWLO) temsili Şekil 3 'te görülmektedir.

VLBI analizinde saat hataları, zenit sslak gecikmeleri, troposfer gradyanları, Yer dönme parametreleri (Earth orientation parameters, EOP), antenlerin TRF ve kuazarların CRF koordinatları sürekli parçalı lineer ofsetler (CPWLO) şeklinde kestirilebilir. $t$ ölçü epoğudaki VLBI gecikme modelinin (ölçünün), $t_{1}$ ve $t_{2}$ kestirim epoklarındaki $x_{1}$ ve $x_{2}$ ofsetlerine (CPWLO) göre kismi türevleri:

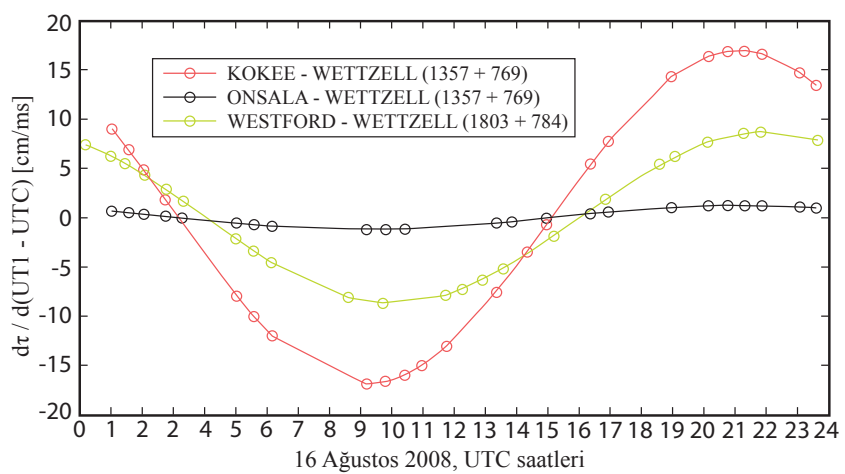

Şekil 2: 16 Agustos 2008 tarihinde gerçekleştirilen 24 saatlik bir VLBI oturumu boyunca üç bazın: Kokee-Wettzell, Onsala-Wettzell, WestfordWettzell iki kutupsal kuazara: 1357+769, 1803+784 gerçekleştirdiği gecikme modelinin (ölçüsünün) UT1 parametresine göre kısmi türevleri.

$$
\begin{gathered}
\frac{\partial \tau(t)}{\partial x_{1}}=\frac{\partial \tau(t)}{\partial x_{i}} \cdot \frac{\partial x_{i}}{\partial x_{1}} \\
\frac{\partial \tau(t)}{\partial x_{2}}=\frac{\partial \tau(t)}{\partial x_{i}} \cdot \frac{\partial x_{i}}{\partial x_{2}},
\end{gathered}
$$

eşitliklerinden elde edilir. Burada, (63) eşitliğinde verilen CPWLO fonksiyonunun birinci ve ikinci ofsetlere, $x_{1}$ ve $x_{2}$ göre k1smi türevleri:

$$
\frac{\partial x_{i}}{\partial x_{1}}=1-\frac{t-t_{j}}{t_{j+1}-t_{j}}
$$

$\frac{\partial x_{i}}{\partial x_{2}}=\frac{t-t_{j}}{t_{j+1}-t_{j}}$

şeklinde elde edilir. CPWLO fonksiyonlarında ölçü epoğu, $t$, kestirim epoklarının içerdiği zaman aralığı içerisinde, diğer bir ifade ile kestirim aralığında olmalıdır $\left(t_{j}<t<t_{j+1}\right.$ koşulu sağlanmalıdır). $t$ ölçü epoğundaki VLBI gecikme modelinin UT1 parametresinin birinci ve ikinci ofsetlerine (CPWLO: $x_{1,2}$ ) göre kısmi türevleri aşağıdaki şekilde elde edilir:

$$
\begin{gathered}
\frac{\partial \tau(t)}{\partial x_{1(U T 1)}}=\frac{\partial \tau(t)}{\partial(U T 1)} \cdot \frac{\partial(U T 1)}{\partial x_{1}}, \\
\frac{\partial \tau(t)}{\partial x_{2(U T 1)}}=\frac{\partial \tau(t)}{\partial(U T 1)} \cdot \frac{\partial(U T 1)}{\partial x_{2}} .
\end{gathered}
$$

(68 ve 69) eşitliklerindeki, $\partial \tau(t) / \partial(U T 1),(44)$ eşitliği ile verilen: $t$ ölçü epoğundaki gecikme modelinin, $\tau(t), U T 1$ 'a göre kısmi türevidir. $\partial(U T 1) / \partial x_{12}$ ise (66 ve 67) eşitlikleri ile verilen $U T 1$ parametresinin birinci ve ikinci ofsetlerine (CPWLO: $x_{1,2}$ ) göre kısmi türevleridir. Benzer şekilde, $t$ ölçü epoğundaki gecikme modelinin birinci VLBI anteni, st 1 TRF koordinatlarından $X$ bileşeni birinci ve ikinci ofsetlerine (CPWLO: $x_{1,2}$ ) göre kısmi türevleri:

$$
\frac{\partial \tau(t)}{\partial x_{1\left(X_{s t 1}\right)}}=\frac{\partial \tau(t)}{\partial X_{s t 1}} \cdot \frac{\partial X_{s t 1}}{\partial x_{1}}
$$

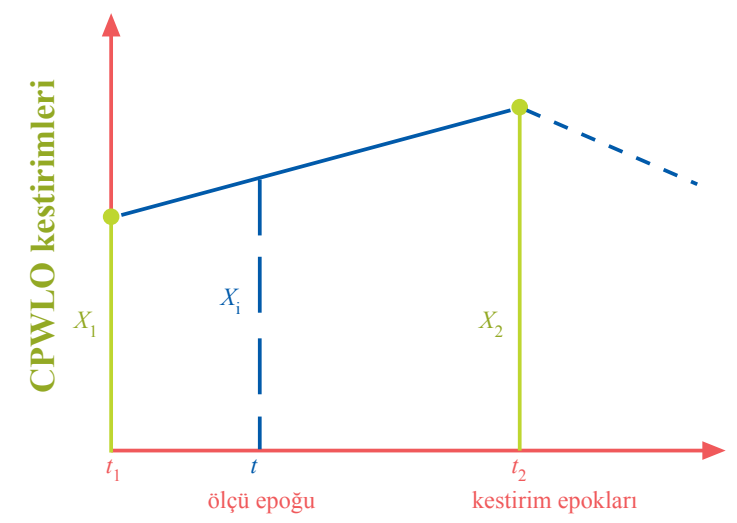

Şekil 3: Sürekli parçalı lineer ofsetler (CPWLO). 
$\frac{\partial \tau(t)}{\partial x_{2\left(X_{s t 1}\right)}}=\frac{\partial \tau(t)}{\partial X_{s t 1}} \cdot \frac{\partial X_{s t 1}}{\partial x_{2}}$.

eşitliklerinden elde edilir. (70 ve 71) eşitliklerindeki, $\partial \tau(t) / \partial X_{s t 1},(61)$ eşitliği ile verilen: $t$ ölçü epoğundaki gecikme modelinin, $\tau(t)$, birinci VLBI anteni, st 1 TRF koordinatlarından $X$ bileşenine göre, $X_{s t 1}$ kısmi türevidir. $\partial X_{s t 1} / \partial x_{1,2}$ ise (66 ve 67) eşitlikleri ile verilen: $X_{s t 1}$ parametresinin birinci ve ikinci ofsetlerine (CPWLO: $x_{1,2}$ ) göre kısmi türevleridir. VLBI ölçülerinden parametre kestiriminde fonksiyonel modele örnek olarak: Üç VLBI anteni ile bir kuazara gerçekleştirilen bir taramadan elde edilen üç adet ölçüyü, $\tau$ (ölçü epoklar1: $t 1^{\prime}, t 2^{\prime}$ ve $\left.t 3^{\prime}\right)$ ve anten TRF koordinatları $X$ bileşenlerinin CPWLO kestirimlerini (bir kestirim aralığı için iki kestirim epoğu olmak üzere her anten için iki adet TRF koordinatı $X$ bileşeni) içeren bir dizayn matrisi aşağıdaki şekilde oluşturulur:

$$
\left[\begin{array}{cccccc}
\frac{\partial \tau\left(t 1^{\prime}\right)}{\partial x_{1\left(X_{s t 1}\right)}} & \frac{\partial \tau\left(t 1^{\prime}\right)}{\partial x_{2\left(X_{s t 1}\right)}} & \frac{-\partial \tau\left(t 1^{\prime}\right)}{\partial x_{1\left(X_{s t 2}\right)}} & \frac{-\partial \tau\left(t 1^{\prime}\right)}{\partial x_{2\left(X_{s t 2}\right)}} & 0 & 0 \\
0 & 0 & \frac{\partial \tau\left(t 2^{\prime}\right)}{\partial x_{1\left(X_{s t 2}\right)}} & \frac{\partial \tau\left(t 2^{\prime}\right)}{\partial x_{2\left(X_{s t 2}\right)}} & \frac{-\partial \tau\left(t 2^{\prime}\right)}{\partial x_{1\left(X_{s t 3}\right)}} & \frac{-\partial \tau\left(t 2^{\prime}\right)}{\partial x_{2\left(X_{s t 3}\right)}} \\
\frac{\partial \tau\left(t 3^{\prime}\right)}{\left.\partial x_{1\left(X_{s t 1}\right)}\right)} & \frac{\partial \tau\left(t 3^{\prime}\right)}{\partial x_{2\left(X_{s t 1}\right)}} & 0 & 0 & \frac{-\partial \tau\left(t 3^{\prime}\right)}{\partial x_{1\left(X_{s t 3}\right)}} & \frac{-\partial \tau\left(t 3^{\prime}\right)}{\partial x_{2\left(X_{s t 3}\right)}}
\end{array}\right]
$$

VLBI ölçülerinin CPWLO gün-içi kestirim parametreleri ile diğer tekniklerden elde edilen parametrelerin karşılaştırılmaS1 amacı ile troposfer zenit toplam gecikmeleri ele alınmıştır. Şekil 4'den görüleceği gibi Wettzell (Almanya) jeodezi yerleşkesindeki VLBI ve GPS tekniklerinden 15 günlük sürekli oturumlardan aynı epoklarda (30 dakika kestirim aralıklı) elde edilen zenit toplam gecikmeleri iyi uyum göstermiştir.
Viyana Teknik Üniversitesi, Jeodezi ve Jeofizik Enstitüsünde geliştirilen Viyana VLBI Yazılımı (Vienna VLBI Software, VieVS) (Böhm vd. 2011a) troposfer zenit toplam gecikmeleri sürekli parçalı lineer ofset (CPWLO) kestirimleri zaman serisi ile Avrupa Yörünge Belirleme Merkezi'nde (Center for Orbit Determination in Europe, CODE) (Dach vd. 2009) gerçekleştirilen Bernese (Dach vd. 2007) GPS troposfer çözümü zaman serisi farklarının \pm 5 mm'lik bir standart sapma değeri ile iyi bir uyum gösterdiği görülmüştür. Yüksek Çözünürlüklü Sınırlı Alan Modeli (The High Resolution Limited Area Model, HIRLAM) (Undén vd. 2002), Avrupa'yı kapsayan bir sayısal atmosfer modeli, diğer uzay jeodezisi teknikleri ile, GPS ve VLBI uyuşumlu (troposfer zenit toplam gecikmeleri serileri fark vektörünün standart sapması: $\pm 15 \mathrm{~mm}$ ) sonuçlar vermiştir.

\section{Sonuçlar}

VLBI ölçülerinden, istasyonlardaki atomik saatlerin hata modeli katsayıları, troposfer zenit gecikmeleri, troposfer gradyanları (troposferin azimutsal asimetrik kısmından ötürü oluşan sinyal gecikmeleri), Yer dönme parametreleri (kutup gezinmesi koordinatları, UT1-UTC, nutasyon ofsetleri), anten TRF referans noktası koordinatları, radyo kaynağı (kuazar) CRF koordinatları parametrelerinin tümü, gün-içi zamansal çözünürlükte, yüksek duyarlıklı ve eş zamanlı kestirilebilir. Bunun için, her parametre kestirim aralığında (iki kestirim epoğu arasında geçen süre) zamansal ve mekansal anlamda homojen dağılmış ve yeterli sayıda VLBI ölçüsü olmalı ve parametre kestirimi aşamasında makalede ayrıntılı olarak ele alındığı üzere sürekli parçalı lineer ofset (CPWLO) fonksiyonu kullanılmalıdır.

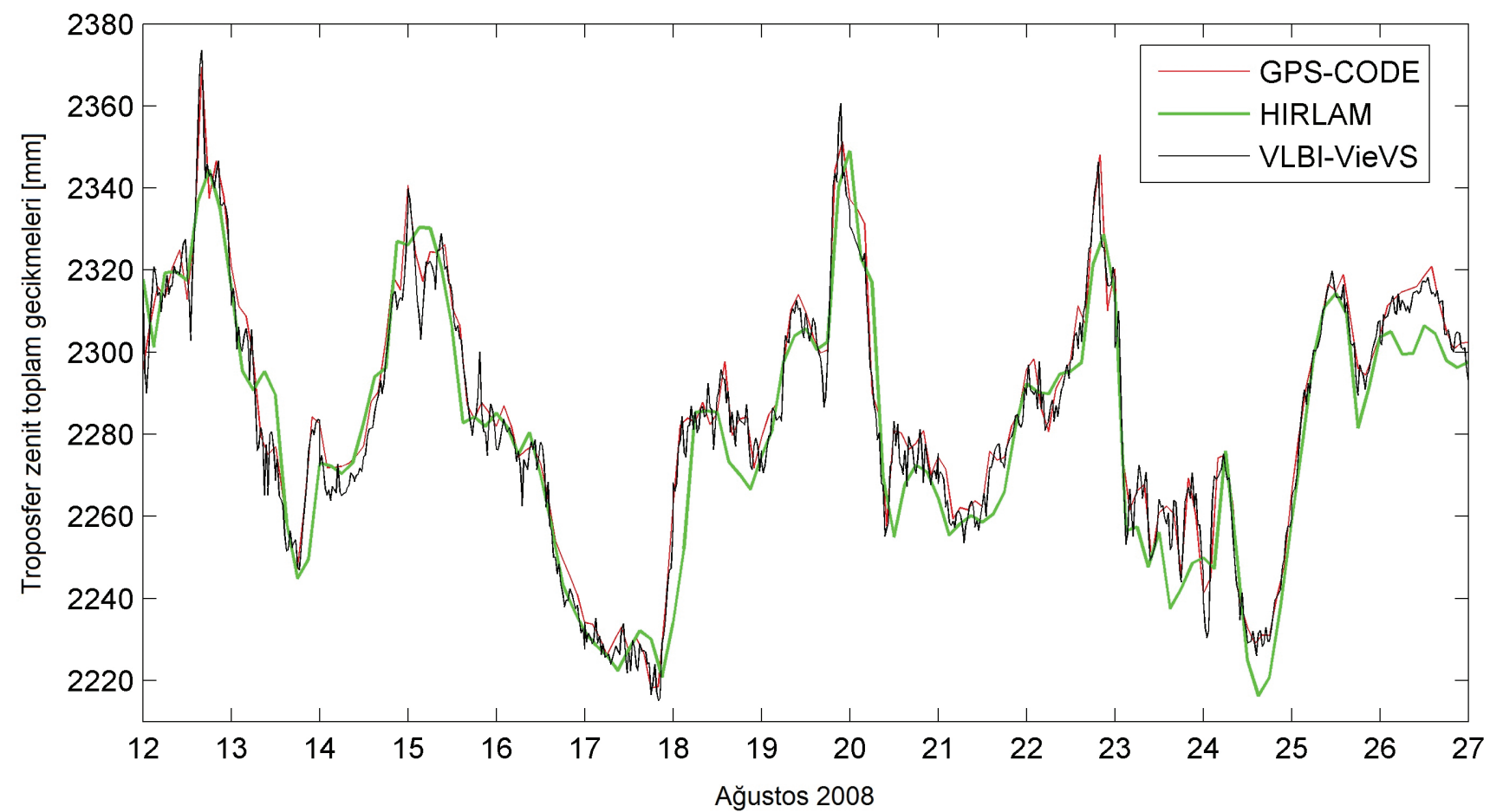

Şekil 4: Wettzell (Almanya) jeodezi yerleşkesinde CONT08 sürekli VLBI oturumları boyunca gün-içi kestirimi yapılan zenit toplam gecikmeleri. CONT08, 12 - 27 Ağustos 2008 tarihlerinde, 5 kıtaya dağılmış, 11 adet jeodezi yerleşkesinde Uluslararası Jeodezi ve Astrometri VLBI Servisi (IVS) tarafından gerçekleştirilen sürekli VLBI oturumlarıdır. GPS-CODE, global IGS analiz merkezlerinden, Avrupa Yörünge Belirleme Merkezi'nde (Center for Orbit Determination in Europe) gerçekleştirilen troposfer çözümüdür. HIRLAM, Yüksek Çözünürlüklü Sınırlı Alan Modeli (The High Resolution Limited Area Model) sayısal atmosfer modeli troposfer ürünüdür. VLBI-VieVS, Viyana Teknik Üniversitesi, Jeodezi ve Jeofizik Enstitüsünde geliştirilen Viyana VLBI Yazılımı'ndan (Vienna VLBI Software) elde edilen troposfer çözümdür. 


\section{Teşekkür}

Bu çalışmada Uluslararası Jeodezi ve Astrometri VLBI Servisi'nin (IVS) sağlamış olduğu ölçü dosyaları kullanılmıştır. Makalenin yazarları bilinmeyen hakemlerden birine yapıcı ve değerli katkılarından ötürü teşekkür ederler.

\section{Kaynaklar}

Böckmann S., Artz T., Nothnagel A., (2010), VLBI terrestrial reference frame contributions to ITRF2008, J. Geod., 84, 201219, doi:10.1007/s00190-009-0357-7.

Böhm J., Böhm S., Nilsson T., Pany A., Plank L., Spicakova H., Teke K., Schuh H., (2011a), The new Vienna VLBI software VieVS, In: International Association of Geodesy Symposia Series, (Kenyon S., Pacino M.C., Marti U., Ed.), Cilt 136, ss. 1007-1013.

Böhm J., Spicakova H., Urquhart L., Steigenberger P., Schuh H., (2011b), Impact of a priori gradients on VLBI-Derived terrestrial reference frames, Proceedings of the $20^{\text {th }}$ Meeting of the European VLBI Group for Geodesy and Astronomy'nin İçinde, (Alef W., Bernhart S., Nothnagel A., Ed.), Institut für Geodäsie und Geoinformation, Universität Bonn, Almanya, Ss.128-132.

Böhm J., Werl B., Schuh H., (2006), Troposphere mapping functions for GPS and Very Long Baseline Interferometry from European Center for Medium-Range Weather Forecasts operational analysis data, J. Geophys. Res., 111(B02406).

Campbell J., (2004), VLBI for Geodesy and Geodynamics, The Role of VLBI in Astrophysics, Astrometry and Geodesy'nin İçinde, (Mantovani F., Kus A., Ed.), Kluwer Academic Publishers, ss.359-381.

Campbell J., (1979), Die Radiointerferometrie auf langen Basen als geodätisches Messprinzip hoher Genauigkeit, DGK Reihe C, Heft 254, Verlag des Instituts für Angewandte Geodäsie, Frankfurt am Main.

Capitaine N., Gambis D., McCarthy D., Petit G., Ray J., Richter B., Rothacher M., Standish M., Vondrak J., (2002), Proceedings of the IERS Workshop on the Implementation of the New IAU Resolutions, IERS Technical Note 29, Verlag des Bundesamts für Kartographie und Geodäsie, Frankfurt am Main.

Capitaine N., Guinot B., McCarthy D., (2000), Definition of the celestial ephemeris origin and of UT1 in the international celestial reference frame, Astron. Astrophys., 335(1), 398-405.

Capitaine N., Guinot B., Souchay J., (1986), A Non-rotating Origin on the Instantaneous Equator: Definition, Properties and Use. Celest. Mech., 39(3), 283-307.

Chen G., Herring T., (1997), Effects of atmospheric azimuthal asymmetry on the analysis from space geodetic data, J Geophys Res, 102 (B9), 20489-20502.

Dach R., Brockmann E., Schaer S., Beutler G., Meindl M., Prange L., Bock H., Jäggi A., Ostini L., (2009), GNSS processing at CODE: status report, J Geod, 83, 353-365.

Dach, R., Hugentobler U., Fridez P., Meindl M., (2007), Bernese GPS Software Version 5.0., Switzerland: Astronomical Institute, University of Bern.

Davis J., Herring T., Shapiro I., (1991), Effects of atmospheric modeling errors on determinations of baseline vectors from VLBI, J. Geophys. Res., 96 (B1), ss. 643-650.

Davis J., Elgered G., Niell A.E., Kuehn C.E., (1993), Ground-based measurements of gradients in the "wet" radio refractivity of air, Radio Sci., 28(6), 1003-1018.
Fey A., Gordon D., Jacobs C.S., (2009), The second realization of the international celestial reference frame by very long baseline interferometry, IERS Technical Note; 35 . Verlag des Bundesamts für Kartographie und Geodäsie, Frankfurt am Main, ISBN 3-89888-918-6, 204 ss.

Herring T., (1992), Modeling atmospheric delays in the analysis of space geodetic data, Proceedings of Refraction of Transatmospheric Signals in Geodesy'nin İçinde, (DeMunck J.C.,Spoelstra T.A., Ed.), Netherlands Geodetic Comission Series, Publications on Geodesy, Cilt.36, ss.157-164.

Herring T., (1986), Precision of vertical estimates from very long baseline interferometry, J. Geophys. Res., 91(B9), 9177-9182.

Lambert S., Bizouard C., (2002), Positioning the Terrestrial Ephemeris Origin in the International Terrestrial Reference Frame, Astron. Astrophys, 394(1), 317-321.

MacMillan D., (1995), Atmospheric gradients from very long baseline interferometry observations, J. Geophys. Res. Lett., 22(9), 1041-1044.

Marini J., (1972), Correction of satellite tracking data for an arbitrary tropospheric profile, Radio Sci., 7(2), 223-231.

Niell A., (1996), Global mapping functions for the atmosphere delay at radio wavelengths, J Geophys. Res., 101(B2), 32273246.

Nothnagel A., (1991), Radiointerferometrische Beobachtungen zur Bestimmung der Polbewegung unter Benutzug langer NordSüd-Basislinien, DGK Reihe C, Heft 368, Verlag des Instituts für Angewandte Geodäsie, Frankfurt am Main.

Petit G., Luzum B., (2010), IERS Conventions 2010, IERS Technical Note ; 36, Frankfurt am Main: Verlag des Bundesamts für Kartographie und Geodäsie, ISBN 3-89888-989-6, 179 ss.

Saastamoinen J., (1972), The use of artificial satellites for geodesy, Geophys. Monogr. Ser.'in İçinde, Cilt 15, ss.247-251, American Geophysical Union, Washington.

Schlüter W., Behrend D., (2007), The International VLBI Service for Geodesy and Astrometry (IVS): current capabilities and future prospects, J. Geod., 81(6-8), 379-387.

Schuh H., (1987), Die Radiointerferometrie auf langen Basen zur Bestimmung von Punktverschiebungen und Erdrotationsparametern, DGK Reihe C, Heft 328, Verlag der Bayerischen Akademie der Wissenschaften, München.

Sovers O.J., Fanselow J.L., Jacobs C.S., (1998), Astrometry and geodesy with radio interferometry: experiments, models, results, Reviews of Modern Physics, 70(4).

Takahashi F., Kondo T., Takahashi Y., Koyama Y., (2000), Wave Summit Course: Very Long Baseline Interferometer, Published by Ohmsha, Ltd., ISBN: 1-58603-076-0 (IOS Press), Tokyo.

Teke K., (2011), Sub-daily parameter estimation in VLBI data analysis, Geowissenschaftliche Mitteilungen, Heft Nr. 87, Vienna University of Technology, Vienna, Austria. Grafisches Zentrum HTU GmbH, ISSN 1811-8380.

Teke K., Böhm J., Nilsson T., Schuh H., Steigenberger P., Dach R., Heinkelmann R., Willis P., Haas R., Espada S.G., Hobiger T., Ichikawa R., Shimizu S., (2011), Multi-technique comparison of troposphere zenith delays and gradients during CONT08, J. Geod., 85(7), 395-413.

Teke K., Boehm J., Tanır E., Schuh H., (2009), Çok Uzun Baz Enterferometrisi (VLBI) tekniğinde, saat hatası ve troposferik gecikme parametre kestirim modelleri, Harita Dergisi, 142, 1-16.

Undén, P., Rontu L., Järvinen H., Lynch P., Calvo J., Cats G., Cuxart J., Eerola K., Fortelius C., Garcia-Moya J., Jones C., Lenderlink G., McDonald A., McGrath R., Navascues B., Woetman N., Ødegaard V., Rodriguez E., Rummukainen M., Room R., Sattler K., Hansen S., Savijärvi H., Wichers S., Sigg R., The H., Tijm A., (2002), HIRLAM-5 Scientific Documentation, Technical report, Swedish Meteorological and Hyrdological Institute, Norrköping. 